\title{
The Abundance of Sulfur Dioxide Below the Clouds of Venus
}

\author{
Bruno Bézard and Catherine de Bergh \\ Département de Recherche Spatiale, Observatoire de Paris, Meudon, France \\ Bruce Fegley
}

Department of Earth and Planetary Sciences and McDonnell Center for the Space Sciences, Washington University, St Louis, Missouri

Jean-Pierre Maillard

Institut d'Astrophysique de Paris, Paris, France

David Crisp

Jet Propulsion Laboratory, Pasadena, California

Tobias Owen

Institute for Astronomy, University of Hawaii, Honolulu, Hawaii

James B. Pollack

NASA/Ames Research Center, Moffett Field, California

David Grinspoon

University of Colorado, Boulder, Colorado

\begin{abstract}
We present a new method for determining the abundance of sulfur dioxide below the clouds of Venus. Absorption by the $3 \nu_{3}$ band of $\mathrm{SO}_{2}$ near $2.45 \mu \mathrm{m}$ has been detected in high-resolution spectra of the night side of Venus recorded at the Canada-France-Hawaii telescope in 1989 and 1991. The inferred $\mathrm{SO}_{2}$ abundance is $130 \pm 40$ ppm at all observed locations and pertains to the 35-45 $\mathrm{km}$ region. These values are comparable to those measured by the Pioneer Venus and Venera 11/12 entry probes in 1978. This stability stands in contrast to the apparent massive decrease in $\mathrm{SO}_{2}$ observed at the cloud tops since these space missions. These results are consistent with laboratory and modelling studies of the $\mathrm{SO}_{2}$ destruction rates in the lower atmosphere of Venus. The new spectroscopic technique presented here allows a remote monitoring of the $\mathrm{SO}_{2}$ abundance below the clouds, a likely tracer of Venusian volcanism.
\end{abstract}

\section{INTRODUCTION}

Sulfur dioxide was first detected above the clouds of Venus by Barker [1979], and later beneath the cloud decks by instruments carried by the Pioneer Venus (PV) and Venera 11/12 entry probes [Oyama et al. 1980, Gel'man et

\section{Copyright 1993 by the American Geophysical Union.}

al. 1979]. $\mathrm{SO}_{2}$ plays important physical and chemical roles in the Venus atmosphere. It is related to the formation of the planet-wide sulfuric acid clouds, the energy budget of the lower atmosphere and greenhouse effect at the surface, and lithosphere-atmosphere interactions such as surface weathering and volcanism.

Telescopic observations show that the night side of Venus emits significant radiation in narrow near-infrared windows located between regions of strong $\mathrm{CO}_{2}$ and $\mathrm{H}_{2} \mathrm{O}$ absorption [Allen and Crawford, 1984]. This emission is thermal radiation originating from the hot lower atmosphere, beneath the sulfuric acid cloud decks. It provides a unique opportunity to study the deep atmosphere of Venus from ground-based observatories. In November 1989, Bézard et al. [1990] recorded the first highresolution $\left(0.28 \mathrm{~cm}^{-1}\right.$ apodized) spectra of the night side of Venus in the 1.7- and 2.3- $\mu \mathrm{m}$ windows. In the latter window, they were able to identify numerous absorption lines due to the gases $\mathrm{CO}_{2}, \mathrm{CO}, \mathrm{H}_{2} \mathrm{O}, \mathrm{HDO}, \mathrm{HF}$, and $\mathrm{COS}$ and measure their abundances in the altitude range probed $(30-50 \mathrm{~km})$. However, Bézard et al. could not reproduce the spectra observed between 4020 and 4100 $\mathrm{cm}^{-1}(2.44-2.49 \mu \mathrm{m})$, and suggested that this difficulty could be due to absorption by the $3 \nu_{3}$ band of $\mathrm{SO}_{2}$, which was not included in the models due to a lack of spectroscopic data at that time. We present an analysis of this spectral region using a recent spectroscopic investigation of the $3 \nu_{3}$ band of $\mathrm{SO}_{2}$ [Lafferty et al., 1992], and derive a new measurement of the abundance of sulfur dioxide below the cloud decks of Venus. 


\section{ObSERVATIONS}

Spectroscopic observations of the night side of Venus at wavelengths near $2.3 \mu \mathrm{m}$ were obtained on 10 November 1989 (UT) with the Fourier Transform Spectrometer at the Canada-France-Hawaii telescope (CFHT) [Bézard et al., 1990]. These spectra were recorded at $0.28 \mathrm{~cm}^{-1}$ resolution (apodized), using a 5-arcsec aperture centered near $30^{\circ} \mathrm{N}$ latitude and $170^{\circ}$ longitude. The angular size of Venus was 25 arcsec. New spectra of the night side were recorded with the same instrument on 28-30 June 1991 UT. The apodized spectral resolution was $0.15 \mathrm{~cm}^{-1}$. Three regions on the dark side centered at $25^{\circ} \mathrm{N}, 5^{\circ} \mathrm{N}$, and $15^{\circ} \mathrm{S}$ (longitude $200^{\circ}$ ), were observed using a 5-arcsec circular aperture, while the angular size of Venus was 28 arcsec. Contamination by reflected sunlight from the day side was removed using dayside spectra recorded right after the nightside spectra. Absolute flux calibration was performed by recording spectra of standard stars at similar zenith angles. The available Venus spectra are displayed in Figure 1.

\section{ANALYSIS}

Several changes have been made to the radiative transfer model used in Bézard et al. [1990]. They are described

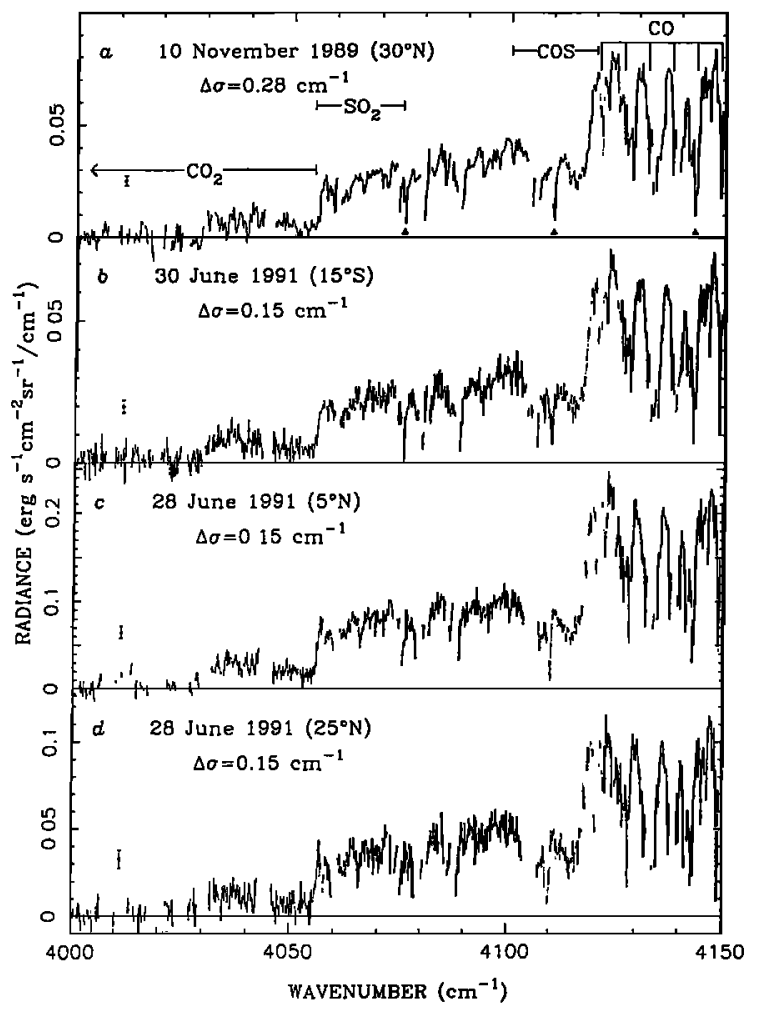

Fig. 1: Spectra of the night side of Venus recorded at $0.28-\mathrm{cm}^{-1}(a)$ and $0.15-\mathrm{cm}^{-1}(b-d)$ resolution. Missing data points correspond to spectral regions where the telluric absorption exceeded $50 \%$. The $\pm 1 \sigma$ level is given for each spectrum. Variations in the cloud optical depth are responsible for the large flux differences observed among the spectra. Absorption features due to $\mathrm{SO}_{2}, \mathrm{COS}, \mathrm{CO}$, $\mathrm{HF}(\boldsymbol{A})$, and $\mathrm{CO}_{2}$ are indicated. All the other absorptions are due to $\mathrm{H}_{2} \mathrm{O}$ and $\mathrm{HDO}$. in detail in Pollack et al. [1993], and are summarized here: (1) We used a new high-temperature $\mathrm{CO}_{2}$ database derived from accurate quantum mechanical calculations. As a result, we can now reproduce the intensity drop observed near $4056 \mathrm{~cm}^{-1}$ due to the head of the weak (01121)(10002) band of ${ }^{12} \mathrm{CO}_{2}$. (2) The hot bands of COS associated with the $2 \nu_{3}$ fundamental have been added to the model. In contrast, only the fundamental mode was included in the modelling of Bézard et al.. (3) Following Pollack et al., we have allowed for a variation in the COS mixing ratio with altitude. These improvements lead to a much better overall agreement between the model and the observed nightside spectra than that achieved previously.

However, there still remains a major discrepancy between observed and synthetic spectra near $4070 \mathrm{~cm}^{-1}$, which is the region of the R-branch of the $3 \nu_{3}$ band of $\mathrm{SO}_{2}$ (Figure 2). Laboratory spectra of this band were recently recorded by two different groups in the U.S. and in France, and line assignments and intensities have been derived [Lafferty et al., 1992]. We used these spectroscopic data to model the $\mathrm{SO}_{2}$ opacity in our atmospheric calculations. We assumed a constant mixing ratio below $48 \mathrm{~km}$, decreasing at higher altitudes (linearly with logarithm of pressure) down to a value of $0.05 \mathrm{ppm}$ at $70 \mathrm{~km}$ [Von Zahn et al., 1983]. Adding $\mathrm{SO}_{2}$ absorption eliminates the previously existing discrepancy and allows us to adequately reproduce the shape of the Venus spectra. A best fit to all of the observed spectra is obtained for a $\mathrm{SO}_{2}$ mixing ratio equal to $130 \pm 40 \mathrm{ppm}$. The error bars encompass instrumental noise as well as uncertainties in the $\mathrm{SO}_{2}$ band intensity, the cloud model, and the $\mathrm{CO}_{2}$ continuum opacity with its possible spectral variations. The atmospheric region sounded by these measurements extends from 35 to $45 \mathrm{~km}$, with a maximum sensitivity near the $39-\mathrm{km}$ level. Our data do not constrain the height variation of $\mathrm{SO}_{2}$; the inferred mixing ratio thus refers to an average over the range of altitudes probed.

\section{Discussion}

Within the mutual uncertainties, our derived $\mathrm{SO}_{2}$ mixing ratio is the same as that measured by the $P V$ and Venera 12 gas chromatographs in December 1978. The PV data yielded $185 \pm 43 \mathrm{ppm}$ at $22 \mathrm{~km}$ altitude [Oyama et al., 1980], while a $\mathrm{SO}_{2}$ mixing ratio of $130 \pm 35 \mathrm{ppm}$ was derived below $42 \mathrm{~km}$ from the Venera 12 measurements [Gel'man et al., 1979]. A recent reanalysis [Bertaux et al., 1992] of the ultraviolet spectra recorded in situ in June 1985 by the scanning spectrophotometers on the Vega 1 and 2 probes yields $120 \pm 20 \mathrm{ppm}$ of $\mathrm{SO}_{2}$ near $42 \mathrm{~km}$, in good agreement with our results.

However, a few measurements subsequent to the PV and Venera 11/12 encounters tend to indicate lower $\mathrm{SO}_{2}$ abundances below the cloud deck at $\sim 48 \mathrm{~km}$. In March 1982, the Venera 13/14 gas chromatograph experiment [Mukhin et al., 1983] detected only small (unquantified amounts) of $\mathrm{SO}_{2}$ and apparently showed that $\mathrm{SO}_{2}$ was less abundant than the reduced sulfur gases COS and $\mathrm{H}_{2} \mathrm{~S}$; but there was a malfunction with the carrier gas flow from this 


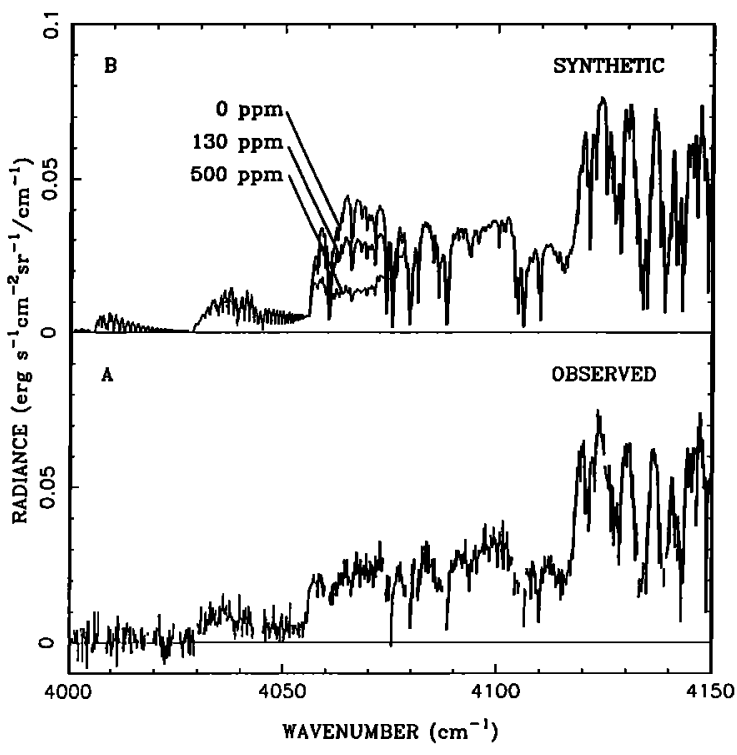

Fig. 2: The observed spectrum $b(A)$ is compared with synthetic spectra $(B)$ in which the subcloud (below 48 $\mathrm{km}) \mathrm{SO}_{2}$ mixing ratio has been varied. Spectra were computed with a $\mathrm{H}_{2} \mathrm{O}$ mixing ratio of $30 \mathrm{ppm}$ below the cloud deck and a constant $\mathrm{HF}$ mixing ratio of $0.006 \mathrm{ppm}$. The CO vertical profile is the one deduced by Von Zahn et al. [1983] from probe data. The COS concentration is $\mathbf{0 . 3 5}$ ppm at $37 \mathrm{~km}$ and increases with depth to a value of 10 ppm at $30 \mathrm{~km}$ and below. The model also includes a continuum opacity $\left(\alpha=4 \times 10^{-8} \mathrm{~cm}^{-1}\right.$ amagat $\left.^{-2}\right)$, needed to reproduce the observed spectrum longward of $4350 \mathrm{~cm}^{-1}$, where $\mathrm{CO}_{2}$ is the only gaseous absorber. The thick curve in $B$ corresponds to the $\mathrm{SO}_{2}$ abundance (130 ppm) which provides the best overall agreement with the observations.

experiment [Krasnopolsky, 1986], and many of its results are suspect. The $\mathrm{SO}_{2}$ profiles retrieved from the Vega 1 and 2 measurements in 1985 strongly decrease downwards below the cloud deck reaching 20-25 ppm at 12 $\mathrm{km}$. The mixing ratios derived at $22 \mathrm{~km}(30-40 \mathrm{ppm})$ are $\sim 5$ times lower than measured by the PV gas chromatograph in 1978. Finally, in March-April 1987, broad-band microwave observations of Venus gave $\mathrm{SO}_{2}$ upper limits as low as $60 \mathrm{ppm}$ below the clouds [Steffes et al., 1990]. However, the microwave spectrum of Venus is not very sensitive to the $\mathrm{SO}_{2}$ opacity and the uncertainties related to errors in the absolute calibration were not taken into account in the analysis.

These spacecraft in situ measurements and groundbased microwave observations show a wide range of values, and have been interpreted to show a monotonic decrease in the $\mathrm{SO}_{2}$ mixing ratio below the clouds since 1978 . Our observations clearly contradict the latter conclusion, as well as the proposal by Wood and Hashimoto [1991] that the $185 \mathrm{ppm}$ of $\mathrm{SO}_{2}$ observed in 1978 was the result of a transient volcanic effusion.

In contrast, UV spectroscopic measurements [Esposito 1984, Esposito et al. 1988, $\mathrm{Na}$ et al. 1990], which probe the Venusian cloud tops, show as much as an order of magnitude decrease in the abundance of $\mathrm{SO}_{2}$ from 1978 to 1988 . The higher $\mathrm{SO}_{2}$ abundance in 1978 was hypothesized to be the result of a major volcanic eruption prior to the PV and Venera missions [Esposito, 1984]. The subsequent drop off would then be due to the conversion of $\mathrm{SO}_{2}$ to $\mathrm{H}_{2} \mathrm{SO}_{4}$ aerosols. Another possibility is that the observed variation results from dynamical and/or photochemical processes in the upper atmosphere. In any case, our observations show that this drop off has no connection to what is happening below the clouds.

In addition, a rapid decline in the $\mathrm{SO}_{2}$ abundance below the clouds is difficult to explain in the framework of existing thermochemical models of the deep atmosphere of Venus. Chemical reactions with surface minerals advocated by some workers [e.g., Wood and Hashimoto, 1991] cannot buffer $\mathrm{SO}_{2}$ at low levels because of the extremely slow reaction rates involved. Laboratory studies of the $\mathrm{SO}_{2}$ reaction rate with $\mathrm{Ca}$-bearing minerals such as calcite, diopside, and anorthite, which are believed to be present on the surface of Venus [Fegley and Treiman 1992, and references therein], show that millions of years are required to remove $\mathrm{SO}_{2}$ from the atmosphere by anhydrite $\left(\mathrm{CaSO}_{4}\right)$ formation [Fegley and Prinn 1989, Fegley and Treiman 1992]. During the intervening 14 years, since the discovery of enhanced cloud-top $\mathrm{SO}_{2}$ abundances, the fastest anhydrite formation reaction $\left(\mathrm{SO}_{2}+\right.$ calcite $)$ would have removed only $0.009 \%$ of the $\sim 150 \mathrm{ppm} \mathrm{SO}_{2}$ observed by PV and Venera 12 in 1978.

We cannot either find any plausible atmospheric sink which could have consumed a large fraction of the $\mathrm{SO}_{2}$ observed in 1978. Calculations of buffering by minerals [Fegley and Treiman, 1992] and of thermochemical equilibrium in the deep atmosphere [Krasnopolsky and Parshev, 1979] do predict COS mixing ratios of a few tens of ppm near the surface, but show that $\mathrm{SO}_{2}$ is still by far the dominant sulfur-bearing gas at low altitudes. COS formation could thus neither have removed much of the $\sim 150$ ppm $\mathrm{SO}_{2}$, nor produce a strong vertical gradient in the $\mathrm{SO}_{2}$ profile as derived from the Vega 1 and 2 spectrophotometers [Bertaux et al., 1992].

Hence, both our observations, and existing models cast doubts on the validity of the $\mathrm{SO}_{2}$ measurements below the clouds taken during the 1980's that indicated monotonically decreasing amounts. They suggest that the $\mathrm{SO}_{2}$ abundance in the deep atmosphere has changed little, if any, since the PV and Venera 12 encounters in 1978.

Spectroscopic observations of the nightside provide a unique means of remotely monitoring the $\mathrm{SO}_{2}$ abundance below the clouds of Venus. These capabilities are crucial for a better understanding of the origin and distribution of $\mathrm{SO}_{2}$ and of the sulfur chemistry on Venus.

Acknowledgments. We are indebted to W.J. Lafferty et al. for diligently carrying out measurements and spectral analysis of the $3 \nu_{3}$ band of $\mathrm{SO}_{2}$. B.F. was supported by grants from the NASA Planetary Atmospheres and Venus Data Analysis Programs. The Canada-FranceHawaii Telescope is operated by the National Research 
Council of Canada, the Centre National de la Recherche Scientifique de France, and the University of Hawaii.

\section{REFERENCES}

Allen, D.A., and J.W. Crawford, Cloud structure on the dark side of Venus, Nature, 307, 222-224, 1984.

Barker, E.S., Detection of $\mathrm{SO}_{2}$ in the UV spectrum of Venus, Geophys. Res. Lett., 6, 117-120, 1979.

Bertaux, J.L., T. Widemann, V.I. Moroz, and A.P. Ekonomov, Vega measurements of $\mathrm{SO}_{2}$ through the atmosphere of Venus: absence of evidence for recent volcanism, Bull. Amer. Astron. Soc., 24, 996, 1992.

Bézard, B., C. de Bergh, D. Crisp, and J.P. Maillard, The deep atmosphere of Venus revealed by high-resolution nightside spectra, Nature, 345, 508-511, 1990.

Esposito, L.W., Sulfur dioxide: episodic injection shows evidence for active Venus volcanism, Science, 223, 10721074, 1984.

Esposito, L.W., M. Copley, R. Eckert, L. Gates, A.I.F. Stewart, and $\mathrm{H}$. Warden, Sulfur dioxide at the Venus cloud tops, 1978-1986, J. Geophys. Res., 93, 5267-5276, 1988.

Fegley, B. Jr., and R.G. Prinn, Estimation of the rate of volcanism on Venus from reaction rate measurements, $\mathrm{Na}$ ture, 397, 55-58, 1989.

Fegley, B. Jr., and A.H. Treiman, Chemistry of atmospheresurface interactions on Venus and Mars, in Venus and Mars: Atmospheres, Ionospheres, and Solar Wind Interactions, pp. 7-71, edited by J. Luhmann \& R. Pepin, AGU, Washington, D.C., 1992.

Gel'man, B.G., V.G. Zolotukhin, N.I. Lamonov, B.V. Levchuk, A.N. Lipatov, L.M. Mukhin, D.F. Nenarokov, V.A. Rotin, and B.P. Okhotnikov, An analysis of the chemical composition of the atmosphere of Venus on an AMS of the Venera-12 using a gas chromatograph, Cosmic Res., 17, 585-589, 1979.

Krasnopolsky, V.A., Photochemistry of the Atmospheres of Mars and Venus, Springer-Verlag, Berlin, 1986.

Krasnopolsky, V.A, and V.A. Parshev, Chemical composition of Venus' troposphere and cloud layer based on Venera 11, Venera 12, and Pioneer Venus measurements, Cosmic Res., 17, 630-637, 1979.

Lafferty, W.J., G.T. Fraser, A.S. Pine, J.-M. Flaud, C. Camy-Peyret, V. Dana, J.-Y. Mandin, A. Barbe, J.-J. Plateaux, and $\mathrm{S}$. Bouazza, The $3 \nu_{3}$ band of ${ }^{32} \mathrm{~S}^{16} \mathrm{O}_{2}$ : line positions and intensities, J. Mol. Spectrosc., 154, 51-60, 1992.

Mukhin, L.M., B.G. Gel'man, N.I. Lamonov, V.V. Mel'ni- kov, D.F. Nenarokov, B.P. Okhotnikov, V.A. Rotin, and V.N. Khokhlov, Gas-chromatograph analysis of the chemical composition of the atmosphere of Venus by the landers of the Venera 13 and Venera 14 spacecraft, Cosmic Res., 21, 168-172, 1983.

$\mathrm{Na}$, C.Y., L.W. Esposito, and T.E. Skinner, International Ultraviolet Explorer observation of Venus $\mathrm{SO}_{2}$ and $\mathrm{SO}, J$. Geophys. Res., 95, 7485-7491, 1990.

Oyama, V.I., G.C. Carle, F. Woeller, J.B. Pollack, R.T Reynolds, and R.A. Craig, Pioneer Venus gas chromatography of the lower atmosphere of Venus, J. Geophys. Res., 85, 7891-7902, 1980.

Pollack, J.B., J.B. Dalton, D. Grinspoon, R.B. Wattson, R, Freedman, D. Crisp, D.A. Allen, B. Bézard, C. De Bergh, L.P. Giver, Q. Ma, and R. Tipping, Near-infrared light from Venus' nightside: a spectroscopic analysis, Icarus, in press, 1993.

Steffes, P.G., M.J. Klein, and J.M Jenkins, Observations of the microwave emission of Venus from 1.3 to $3.6 \mathrm{~cm}$, Icarus, 84, 83-92, 1990.

Von Zahn, U., S. Kumar, H. Niemann, and R. Prinn, Composition of the Venus atmosphere, in Venus, edited by D.M. Hunten, L. Colin, T.M Donahue, \& V.I Moroz, pp. 299-430, University of Arizona Press, Tucson, 1983.

Wood, J.A., and A. Hashimoto, Weathering on Venus: Dependence of mineralogy on altitude and atmospheric composition, Lunar Planet. Sci., Vol. XXII, 1519-1520, 1991.

Bruno Bézard and Catherine de Bergh, Département de Recherche Spatiale, Observatoire de Paris (Section de Meudon), 92195 Meudon Principal Cedex, France

Bruce Fegley, Department of Earth \& Planetary Sciences and McDonnell Center for the Space Sciences, Washington University, St. Louis MO 63130-4899

Jean-Pierre Maillard, Institut d'Astrophysique de Paris, 98 $8^{\text {bis }}$ Boulevard Arago, 75014 Paris, France

David Crisp, Jet Propulsion Laboratory, MS 169-237, 4800 Oak Grove Drive, Pasadena, CA 91109

Tobias Owen, Institute for Astronomy, University of Hawaii, 2680 Woodlawn Drive, Honolulu, HI 96822

James B. Pollack, NASA/Ames Research Center, MS 245-3, Moffett Field, CA 94035

David Grinspoon, University of Colorado, Boulder, CO 80309

(Received December 21, 1992; Revised May 5, 1993;

Accepted May 21, 1993) 